\title{
Treatment of medulloblastoma in children and adolescents
}

\author{
TRATAMENTO DO MEDULOBLASTOMA EM CRIANÇAS E ADOLESCENTES \\ Authorship: Sociedade Brasileira de Neurocirurgia \\ Participants: Nelci Zanon Collange ${ }^{1}$, Silvana de Azevedo Brito ${ }^{1}$, Ricardo Rezende Campos ${ }^{1}$, \\ Egmond Alves Silva Santos ${ }^{1}$, Ricardo Vieira Botelho ${ }^{1}$ \\ Conflict of interest: none
}

${ }^{1}$ Sociedade Brasileira de Neurocirurgia

The Guidelines Project, an initiative of the Brazilian Medical Association, aims to combine information from the medical field in order to standardize procedures to assist the reasoning and decision-making of doctors.

The information provided through this project must be assessed and criticized by the physician responsible for the conduct that will be adopted, depending on the conditions and the clinical status of each patient.

\section{INTRODUCTION}

Medulloblastoma is the most common brain tumor in children from zero to four years old. It is rare in adults and $75 \%$ of cases occur in patients younger than 16 years. ${ }^{1}$

Treatment includes surgery, chemo and radiotherapy. Controversy persists about the need for full withdrawal, need and indication of reoperation for residual lesion, the role of neuraxial chemotherapy and radiotherapy, and best treatment of hydrocephalus, which often accompanies these tumors.

This guideline is addressed to physicians, health managers and decision makers in the treatment of this disease.

The guideline was based on clinical issues that were specified for the type of patient, and the method of development for this guideline included the databases that were searched, the electronic search strategy, types of study under evaluation, and the criteria used to produce recommendation. The clinical issues were defended by the authors (group of neurosurgeons of the Pediatric Neurosurgery Department, Sociedade Brasileira de Neurocirurgia - Brazilian Society of Neurosurgery). The electronic search strategies for each clinical question are described in Annex I. The number after each strategy reveals the number of articles initially retrieved by the strategy. Regarding the extraction of data, items ultimately identified as related to the issue were described in the text. The others were excluded because they did not specifically address the issue.

\section{Objective}

To define the best alternatives for diagnosis and treatment of medulloblastoma in children and adolescents, and recommend them where possible.

\section{Method}

Patients for whom the guideline was constructed are children, adolescents and young adults aged between 0-18 years with diagnosis of medulloblastoma.

The methods for developing this guideline were those involved in evidence-based medicine, establishing primary trials as the studies with the best quality of existing information for decision making.

Databases searched included the Medline, 1966 to 2013, and the Central Cochrane Register of Controlled Trials, using the terms of the standard vocabulary (MESH) and terms from the text: The references obtained in the primary studies were evaluated in search of other work, as well as works of knowledge of the authors of the guideline.

The correspondence between the degree of recommendation and the strength of scientific evidence is described after extracting data of each work (in parentheses), and was classified as follows:

A. Experimental or observational studies of higher consistency.

B. Experimental or observational studies of lower consistency.

C. Case reports and non-controlled studies.

D. Opinions without critical evaluation, based on consensus, physiological studies, or animal models.

Size and direction of the effect, benefits, side effects and risks were considered to develop these recommendations.

Articles were selected according to the hierarchy of evidence produced, with priority given to randomized trials and, in their absence, those with highest level of evidence available. 


\section{Chinical questions}

\section{What is the Natural history OF MEdullo- BLASTOMA IN CHILDREN AND ADOLESCENTS?}

The search strategy is described in Annex I. There are few articles describing its natural history. The electronic search found 22 publications, whose final evaluation revealed two papers describing the natural history in adults and two in children. Three other works derived from the study of references and knowledge of the authors.

\section{Data extraction}

- Importance of the age: Among children and adolescents with medulloblastoma, children under three years of age have worse prognosis than those older than that. ${ }^{3}$

- Degree of tumor differentiation: The degree of differentiation of tumor tissue is important for survival. In one study, patients with $90 \%$ or more of undifferentiated total tumor tissue exhibited extremely poor prognosis (with none of the nine patients operated remaining alive after 3 years) in contrast with $60 \%$ survival in patients with tumors that showed levels of differentiation below $90 \%{ }^{3}$ (C)

- Disease-free interval after treatment: After treatment, disease-free survival at 5 years reached $54 \%$ in one study. ${ }^{2}$ (C) Tumor recurrence, if any, occurs before eight years of treatment.

Severity of hydrocephalus, size of the tumor, and presence of brain-stem invasion were not correlated with prognosis. ${ }^{4}(\mathrm{D}),{ }^{5}(\mathrm{C}),{ }^{6}(\mathrm{C})(\mathrm{C})$

\section{Recommendation}

It is recommended to consider the medulloblastoma as serious illness. In children under 3 years with undifferentiated tumors, survival is lower. Children older than 3 years with less anaplastic tumors have better survival rates. (C)

\section{IS COMPLETE RESECTION OF MEDULLOBLASTOMA REQUIRED?}

Studies comparing partial and complete resections were evaluated. The search strategy was described in Annex I. 960 articles were found from the electronic search. Of these, 12 articles were initially selected based on the title. Seven articles were selected based on summaries. The Solheim article was excluded as it describes comparisons of mortality among regions in the same country.

\section{Data extraction}

Lang et al. ${ }^{7}$ (C) published a series of cases with retrospective analysis in children older than six months with brain tumor. There were four patients with primitive neuroectodermal tumors/medulloblastoma. There were two intraoperative deaths among 16 cases operated. Of the 14 survivors, four had total resections. One patient with medulloblastoma underwent complete resection (>90\%) and was treated with chemotherapy and radiotherapy. Survival lasted 1.2 years and, in terms of neurological status, psychomotor development was impaired, the child was not able to crawl or stand, could speak a few words, and did not present neuropsychological improvements during development. Another patient was operated at the age of 1.4 months, survived for six months and underwent ventriculoperitoneal shunt as adjunctive therapy only. The authors describe four cases of medulloblastoma in the series but the result of only two of the patients.

Raimondi and Tomita ${ }^{8}$ (C) studied 22 patients. Complete resection was performed in 13 patients, while nine underwent subtotal resection. By then, the patients were studied by CT scans and myelogram. All the patients treated with complete resection were alive at the time when these results were published, as well as 3 out of 9 who underwent partial resection. They all underwent radiotherapy.

Wang et al. ${ }^{9}$ (C) conducted a retrospective study of 52 patients for analysis of prognostic factors. The results of univariate analysis showed that the greater the extent of resection, the better the prognosis. In multivariate analysis, the extent of resection had no significance.

Monteight et al. ${ }^{10}$ (C) studied a series of 166 cases of brain tumor in children. Thirty-six cases were medulloblastoma and were sorted according to the extent of resection: Complete resection, 16 cases; incomplete resection, 14 cases; biopsy, six cases. Patients with complete resection of medulloblastoma had higher survival than those undergoing incomplete resection and/or biopsy.

\section{Recommendation}

There is evidence (C) that complete resection improves survival. Complete resection is recommended wherever possible.

\section{When does the Residual tUMOR, IN ME- DULLOBLASTOMA, NEED TO BE OPERATED AFTER THE FIRST SURGERY?}

124 articles potentially related to our research were identified. Ten were initially selected based on title and summary ${ }^{10-20}$ No article specifically investigated the effect of reoperation on residual tumor. 
The effect of reoperation in case of residual tumor after surgery needs to be studied.

\section{What is the incidence of hydrocephalus IN MEDULLOBLASTOMA?}

Due to the fact that different types of tumor, presenting medial or lateral location, produce different incidences of hydrocephalus and different outcomes in terms of relief of cerebrospinal fluid flow blockage after tumor resection, we only used studies that exclusively addressed medulloblastomas or allowed evaluating the data separately for each type of tumor.

\section{Data extraction}

Kombogiorgas, ${ }^{21}$ Hoffman ${ }^{22}$ and Muzundar ${ }^{23}$ studied specific series of medulloblastoma. Muzundar, in a series of 154 patients (2001-2010), found hydrocephalus in $96.5 \%$ of cases. Komborgiorgas, in 2008 , found $100 \%$ of hydrocephalus in 20 patients, while Hoffman, in a series of 44 patients, observed hydrocephalus in $93.18 \%$ of patients. The three studies yielded an average of $96.56 \%$ of hydrocephalus in patients with medulloblastoma. (C)

\section{What is the effect of tumor Resection FOR THE RESOLUTION OF HYDROCEPHALUS?}

- Due-Tønnessen and Helseth ${ }^{24}$ needed cerebrospinal fluid shunt in 53\% of resected medulloblastomas. (C)

- Kombogiorgas ${ }^{21}$ in 2008, operated 20 patients with medulloblastoma with 4 requiring shunt (20\%). (C)

- Gopalakrishnan el al. ${ }^{25}$ showed that $36.8 \%$ of 38 patients with medulloblastoma required CSF shunt. (C)

- Kumar et al. ${ }^{26}$ showed that $22.72 \%$ of their patients needed post-operative shunt. Seven out of the 55 patients undergoing total resection required CSF shunt (12.7\%), compared with 13 of the 33 treated with partial resection (39.3\%). (C)

- Moreelliir had 6 out of 27 patients operated for medulloblastoma (22.22\%) requiring CSF shunt. (C)

- Lee et al. ${ }^{28}$ studied 42 patients and found a need for CSF shunt in 17 (40\%). (C)

The average need for CSF shunt after tumor resection was $32.45 \%$ (20 to $53 \%$ ). Tumor resection treats the associated hydrocephalus in $67.55 \%$ of cases. (C)

\section{Recommendation}

Tumor removal is recommended for the treatment of hydrocephalus. (C)

\section{What is the efFect of Endoscopic thiRd VENTRICULOSTOMY IN HYDROCEPHALUS ASSOCIA- TED WITH MEDULLOBLASTOMA COMPARED WITH VENTRICULOPERITONEAL SHUNT?}

The electronic search is described in Annex I. 64 studies were retrieved, but only one by El-Ghandour ${ }^{29}$ (B) compared endoscopic third ventriculostomy (ETV) and ventriculoperitoneal shunt (VPS) in the treatment of pediatric patients with severe obstructive hydrocephalus due to tumors in the posterior fossa in 53 cases (32 medulloblastomas).

\section{Data extraction}

In the third-ventriculostomy group, complications occurred in three patients (two intraoperative bleeding, and one case of cerebrospinal fluid leaks), while the CSF shunt group, complications occurred in six patients (shunt infection in two cases, with one death; subdural collection in two cases; epidural hematoma in one case; and upward herniation in one case). Endoscopic third ventriculostomy required less surgical time compared with ventriculoperitoneal shunt (15 min vs. $35 \mathrm{~min}$ ).

Data from a single non-randomized study with no major differences in complications between the groups ( 6 vs. 3) do not allow us to assert the superiority of one method or another.

\section{Recommendation}

Third-ventriculostomy and ventriculoperitoneal shunt are options in the treatment of hydrocephalus persisting after surgical removal of medulloblastoma.

\section{The USE OF RADIOTHERAPY IS NEEDED IN ME- DULLOBLASTOMA?}

A study by Bouffet ${ }^{30}$ (C), performed in 1992, postponed radiation only in the supratentorial area and kept $\mathrm{RxT}$ in the posterior fossa and spinal cord. Patients had relapsed medulloblastoma in the supratentorial area. Based on this study, radiation therapy has been performed in medulloblastoma to prevent recurrence. ${ }^{33-35}$

\section{Recommendation}

Supra- and infratentorial radiotherapy is recommended to treat children with medulloblastoma (aged 3 years or younger). (B)

\section{ShOUld RADiotherapy be Given to ChIL- DREN UNDER 3 YEARS?}

Lafay-Cousin et al. ${ }^{31}$ (B) studied 29 patients with medulloblastoma aged three years or younger, comparing the 
use of conventional craniospinal radiotherapy (group treated before 1994) with radiation therapy only in case of relapse or disease progression. The group receiving conventional radiotherapy showed significant lowering of intellectual and academic performance compared with the group in which radiotherapy was delayed. Radiotherapy has been avoided in children under 3 years old. ${ }^{32}$

\section{Recommendation}

Radiotherapy should be avoided or used only in cases of relapse or lack of tumor control in order to prevent intellectual deficit. (B)

\section{AnNex I: SEARCh STRATEgIES AND NUMBER OF STUDIES INITIALLY RETRIEVED FROM THE ELECTRO- NIC SEARCH}

Search strategy: Natural history

- ("medulloblastoma" [MeSH Terms] OR "medulloblastoma" [All Fields]) AND ("natural history" [MeSH Terms] OR ("natural” [All Fields] AND "history" [All Fields]) OR "natural history" [All Fields]) - 22.

\section{Search strategy: Effect of the extension of tumor resection}

- ("medulloblastoma" [MeSH Terms] OR "medulloblastoma” [All Fields]) AND ((“craniotomy" [MeSH Terms] OR "craniotomy" [All Fields]) OR ("microsurgery" [MeSH Terms] OR "microsurgery" [All Fields]) OR ("neurosurgical procedures" [MeSH Terms] OR ("neurosurgical" [All Fields] AND "procedures" [All Fields]) OR "'neurosurgery”[All Fields] OR "neurosurgery" [MeSH Terms]) OR ("neurosurgical” [All Fields] AND "procedures" [All Fields]) OR "neurosurgical procedures" [All Fields])) - 960.

Search strategy: Reoperation in the presence of residual tumor after surgery

- ("medulloblastoma" [MeSH Terms] OR "medulloblastoma” [All Fields]) AND ("residual tumour" [All Fields] OR "neoplasm, residual" [MeSH Terms] OR ("neoplasm" [All Fields] AND "residual” [All Fields]) OR "residual neoplasm" [All Fields] OR ("residual” [All Fields] AND "tumor" [All Fields]) OR "residual tumor" [All Fields]) -124.

Search strategy: Comparison of tumor removal, third-ventriculostomy, and CSF shunt to treat hydrocephalus

- ("medulloblastoma" [MeSH Terms] OR "medulloblastoma” [All Fields]) AND ("hydrocephalus" [MeSH Terms] OR "hydrocephalus" [All Fields]) AND "man- agement" [All Fields] OR "disease man-agement" [MeSH Terms] OR ("disease" [All Fields] AND "management" [All Fields]) OR "disease management" [All Fields]) OR ("therapy" [Subheading] OR "therapy" [All Fields] OR "treatment" [All Fields] OR "therapeutics" [MeSH Terms] OR "therapeutics" [All Fields])) - 159 .

Search strategy: What is the effect of endoscopic third ventriculostomy in hydrocephalus associated with medulloblastoma compared with ventriculoperitoneal shunt?

- ("medulloblastoma" [MeSH Terms] OR "medulloblastoma” [All Fields]) AND ("endoscopic third ventriculostomy" [All Fields] OR "ventriculoperitoneal shunt" [All Fields]) -64.

Search strategy: Is it necessary to use radiotherapy in medulloblastoma?

- "Medulloblastoma/radiotherapy" [Mesh] AND ("randomized controlled trial" [Publication Type] OR "randomized controlled trials as topic" [MeSH Terms] OR "randomized controlled trial" [All Fields] OR "randomised controlled trial" [All Fields]) - 129.

Search strategy: What is the effect of radiotherapy in children under 3 years old?

- ("medulloblastoma" [MeSH Terms] OR "medulloblastoma" [All Fields]) AND ("radiotherapy" [Subheading] OR "radiotherapy" [All Fields] OR "radiotherapy" [MeSH Terms]) AND younger [All Fields] AND ("child" [MeSH Terms] OR "child" [All Fields] OR "children" [All Fields]) -76.

\section{References}

1. Smee RI, Williams JR, De-Loyde KJ, Meagher NS, Cohn R. Medulloblastoma: progress over time. J Med Imaging Radiat Oncol. 2012; 56(2):227-34.

2. Albright AL, Wissof JH, Zeltzer PM, Boyett JM, Rorke LB, Stanley P. Effects of medulloblastoma ressections on outcome in children: a report from the Children's Cancer Group. Neurosurgery. 1996; 38(2):265-71.

3. Gaffney CC, Sloane JP, Bradley NJ, Bloom HJ. Primitive neuroectodermal tumours of the cerebrum. Pathology and treatment. J Neurooncol. 1985; 3(1):23-33.

4. Muraszko KM, Brahma B, Orringer D. Medulloblastoma. In: Albright AL, Pollack IF, Adelson PD. Principles and practice of pediatric neurosurgery. New York: Thieme, 2008. p.613.

5. Kunschner LJ, Kuttesch J, Hess K, Yung WK. Survival and recurrence factors in adult medulloblastoma: the M.D. Anderson Cancer Center experience from 1978 to 1998. Neuro Oncol. 2001; 3(3):167-73.

6. Boiardi A, Silvani A, Eoli M, Fariselli L, Zappacosta B, Salmaggi A. Embryonal tumors in the adult population: implications in therapeutic planning. Neurol Sci. 2000; 21(1):23-30.

7. Lang SS, Beslow LA, Gabel B, Judkins AR, Fisher MJ, Sutton LN, et al. Surgical treatment of brain tumors in infants younger than six months of age and review of the literature. World Neurosurg. 2012; 78(1-2):137-44.

8. Raimondi AJ, Tomita T. Medulloblastoma in childhood: comparative results of partial and total resection. Childs Brain. 1979; 5(3):310-28. 
9. Wang KC, Lee JI, Cho BK, Kim IH, Kim JY, Shin HY, et al. Treatment outcome and prognostic factors of medulloblastoma. J Korean Med Sci. 1994; 9(1):64-73.

10. Monteith SJ, Heppner PA, Woodfield MJ, Law AJ. Paediatric central nervous system tumours in a New Zealand population: a 10-year experience of epidemiology, management strategies and outcomes. J Clin Neurosci. 2006; 13(7):722-9.

11. Fried I, Huang A, Bartels U, Tabori U, Laperriere N, Dirks P, et al. Chronic residual lesions in metastatic medulloblastoma patients. J Pediatr Hematol Oncol. 2014; 36(1):71-5

12. Kombogiorgas D, Puget S, Boddaert N, Peet A, English M, Natarajan K, et al. Appraisal of the current staging system for residual medulloblastoma by volumetric analysis. Childs Nerv Syst. 2011;27(12):2101-6.

13. Ranger A, McDonald W, Bauman GS, Del Maestro R. Effects of surgical excision and radiation on medulloblastoma cell invasiveness. Can J Neurol Sci. 2009; 36(5):631-7.

14. Schubert MI, Wilke M, Müller-Weihrich S, Auer DP. Diffusion-weighted magnetic resonance imaging of treatment-associated changes in recurrent and residual medulloblastoma: preliminary observations in three children. Acta Radiol. 2006; 47(10):1100-4.

15. Zeltzer PM, Boyett JM, Finlay JL, Albright AL, Rorke LB, Milstein JM, et al. Metastasis stage, adjuvant treatment, and residual tumor are prognostic factors for medulloblastoma in children: conclusions from the Children's Cancer Group 921 randomized phase III study. J Clin Oncol. 1999; 17(3):832-45.

16. Albright AL, Wisoff JH, Zeltzer PM, Boyett JM, Rorke LB, Stanley P. Effects of medulloblastoma resections on outcome in children: a report from the Children's Cancer Group. Neurosurgery. 1996; 38(2):265-71.

17. Sure U, Berghorn WJ, Bertalanffy H, Wakabayashi T, Yoshida J, Sugita K, et al. Staging, scoring and grading of medulloblastoma. A postoperative prognosis predicting system based on the cases of a single institute. Acta Neurochir (Wien). 1995; 132(1-3):59-65

18. Patrice SJ, Tarbell NJ, Goumnerova LC, Shrieve DC, Black PM, Loeffler JS. Results of radiosurgery in the management of recurrent and residual medulloblastoma. Pediatr Neurosurg. 1995; 22(4):197-203.

19. Bourne JP, Geyer R, Berger M, Griffin B, Milstein J. The prognostic significance of postoperative residual contrast enhancement on CT scan in pediatric patients with medulloblastoma. J Neurooncol. 1992; 14(3):263-70.

20. Korah MP, Esiashvili N, Mazewski CM, Hudgins RJ, Tighiouart M, Janss AJ, et al. Incidence, risks, and sequelae of posterior fossa syndrome in pediatric medulloblastoma. Int J Radiat Oncol Biol Phys. 2010; 77(1):106-12.

21. Kombogiorgas D, Natarajan K, Sgouros S. Predictive value of preoperative ventricular volume on the need for permanent hydrocephalus treatment immediately after resection of posterior fossa medulloblastomas in children. J Neurosurg Pediatr. 2008; 1(6):451-5.

22. Hoffman HJ, Hendrick EB, Humphreys RP. Metastasis via ventriculoperitoneal shunt in patients with medulloblastoma. J Neurosurg. 1976; 44(5):562-6.
23. Muzumdar DP. Ventricular CSF drainage and medulloblastoma. Ped Neurosurg. 2007; 43(1):74-5.

24. Due-Tønnessen BJ, Helseth E. Management of hydrocephalus in children with posterior fossa tumors: role of tumor surgery. Pediatr Neurosurg. 2007; 43(2):92-6.

25. Gopalakrishnan CV, Dhakoji A, Menon G, Nair S. Factors predicting the need for cerebrospinal fluid diversion following posterior fossa tumor surgery in children. Pediatr Neurosurg. 2012; 48(2):93-101.

26. Kumar V, Phipps K, Harkness W, Hayward RD. Ventriculo-peritoneal shunt requirement in children with posterior fossa tumours: an 11-year audit. $\mathrm{Br}$ J Neurosurg. 1996; 10(5):467-70.

27. Morelli D, Pirotte B, Lubansu A, Detemmerman D, Aeby A, Fricx C, et al. Persistent hydrocephalus after early surgical management of posterior fossa tumors in children: is routine preoperative endoscopic third ventriculostomy justified? J Neurosurg. 2005; 103(3 Suppl):247-52.

28. Lee M, Wisoff JH, Abbott R, Freed D, Epstein FJ. Management of hydrocephalus in children with medulloblastoma: prognostic factors for shunting. Pediatr Neurosurg. 1994; 20(4):240-7.

29. El-Ghandour NM. Endoscopic third ventriculostomy versus ventriculoperitoneal shunt in the treatment of obstructive hydrocephalus due to posterior fossa tumors in children. Childs Nerv Syst. 2011; 27(1):117-26.

30. Bouffet E, Bernard JL, Frappaz D, Gentet JC, Roche H, Tron P, et al. M4 protocol for cerebellar medulloblastoma: supratentorial radiotherapy may not be avoided. Int J Radiat Oncol Biol Phys. 1992; 24(1):79-85.

31. Lafay-Cousin L, Bouffet E, Hawkins C, Amid A, Huang A, Mabbott DJ. Impact of radiation avoidance on survival and neurocognitive outcome in infant medulloblastoma. Curr Oncol. 2009; 16(6):21-8.

32. Rutkowski S, Gerber NU, von Hoff K, Gnekow A, Bode U, Graf N, et al.; German Pediatric Brain Tumor Study Group. Treatment of early childhood medulloblastoma by postoperative chemotherapy and deferred radiotherapy. Neuro Oncol. 2009; 11(2):201-10.

33. Lannering B, Rutkowski S, Doz F, Pizer B, Gustafsson G, Navajas A, et al. Hyperfractionated versus conventional radiotherapy followed by chemotherapy in standard-risk medulloblastoma: results from the randomized multicenter HIT-SIOP PNET 4 trial. J Clin Oncol. 2012; 30(26):3187-93.

34. Christopherson KM, Bradley JA, Rotondo RL, Pincus DW, Fort JA, Morris $\mathrm{CG}$, et al. Local control in non-metastatic medulloblastoma. Acta Oncol. 2014; 3:1-7.

35. Pichandi A, Ganesh KM, Jerrin A, Balaji K, Sridhar PS, Surega A. Cranio spinal irradiation of medulloblastoma using high precision techniques - A dosimetric comparison. Technol Cancer Res Treat. 2014. [Epub ahead of print] 